Rischmoller L., Dong N., Fischer M., and Khanzode A. (2017). "Improving On-site Meeting Efficiency by Using and Automated Model Breakdown Tool” In: LC3 2017 Volume II - Proceedings of the 25th Annual Conference of the International Group for Lean Construction (IGLC), Walsh, K., Sacks, R., Brilakis, I.

(eds.), Heraklion, Greece, pp. 397-404. DOI: https://doi.org/10.24928/2017/0217

\title{
IMPROVING ON-SITE MEETING EFFICIENCY BY USING AN AUTOMATED MODEL BREAKDOWN TOOL
}

\author{
Leonardo Rischmoller ${ }^{1}$, Ning (Tony) Dong ${ }^{2}$, Martin Fischer ${ }^{3}$, and Atul Khanzode ${ }^{4}$
}

\begin{abstract}
Integrating Building Information Modelling (BIM) to on-site meetings in the construction phase has been a challenge to general contractors mainly due to the inflexibility of the current practice in grouping and organizing models to facilitate rapid, on-the-spot model queries. We have developed an automation tool that supports the automatic grouping of models according to a certain model breakdown structure (MBS), which reflects a breakdown structure of a project. This enables a model navigator to identify the relevant model contents on the fly in a meeting thus drastically reducing the coordination latency in resolving an issue. This paper introduces two case studies to explain a non-intrusive process of introducing federated models produced by the automation tool to the meetings. The results of surveys to the meeting participants prove that the non-intrusive process as well as the models reflecting multiple MBS's changed the meeting participants' behaviour and enhanced their coordination, communication, and collaboration, thus improving the overall outcomes of the meetings.
\end{abstract}

Keywords: Building Information Model (BIM), Model Breakdown Structure (MBS), Coordination Latency

\section{INTRODUCTION}

Chachere et al. (2004) defines the term "coordination latency" as the time that elapses between a request for information or action and the compliance to that request. Coordination latency is offered as a unifying, intuitive, descriptive performance metric, intended to reach a near-zero value as a project design goal (Chachere et al., 2009). Coordination latency is especially important in the interdependent and iterative design because it involves a large number of information exchanges and exception handling requests (Cachere et al., 2004).

Reduction of coordination latency can lead to shorter design development times, timely availability of critical information, ability to share and communicate useful information on a timely basis, and hence to a transformed near real-time decision-making process in which a number of issues are solved on the spot rather than going through more traditional prolonged processes, such as the request for information process and the change order process.

The introduction of BIM to the design and coordination processes in the AEC industry has transformed design meetings to integrated concurrent engineering (ICE) sessions (Coffee, 2006), in which the coordination latency has been drastically reduced, resulting in extraordinarily rapid design with a quality similar to or surpassing traditional methods at a lower cost (Smith 1998; Smith and Koenig 1998; Wall 1999, Wall 2000; Wall et al., 1999; Kunz and Fischer, 2012). However, bringing BIM to on-site meetings, such as the

DPR BIM Manager, DPR Construction LeonardoR@dpr.com

DPR R\&D Manager TonyD@dpr.com

Center for Integrated Facility Engineering, Stanford University, Director Fischer@stanford.edu

DPR Technology Group Director AtulK@dpr.com 
daily subcontractor huddle, pull planning meetings (Tommelein, 1998), and ownerarchitect-contractor (OAC) meetings has been very challenging. One of the main reasons is that the current practice of issue discussion and resolution using federated models heavily relies on saved viewpoints or snapshots prepared in advance to support these meetings, in which participants usually have many on-the-spot model queries. If a model navigator is not fast enough to identify the right model contents, the field personnel (e.g., superintendents, subcontractors, field engineers, foremen, etc.) will lose interest and stop trusting the use of BIM in these meetings. Current BIM models/processes have too much coordination latency to support real-time dynamic of the meetings and the current BIM practice does not provide adequate support to the reduction of coordination latency in the on-site meetings. Therefore, the full benefits of BIM are not fully reaped in the construction phase. In the paper titled "automation of the building information model breakdown structure" Rischmoller et al. (2017) introduced the CIFE ${ }^{5}$ Model Breakdown Structure (MBS) automation tool and method that enable project participants without extensive BIM trainings the rapid identification of the desired model contents. In this paper, we use a case study approach to demonstrate how the utilization of this tool and method improves the field personnel's involvements with BIM thus improving the efficiencies of the on-site meetings.

\section{BENEFITS OF USING BIM IN THE ON-SITE MEETINGS}

The following are the expected benefits of applying the CIFE MBS automation tool and method to the case studies:

- Improvement (among the meeting participants) of:

- Coordination

- Communication

- Collaboration

- Reduction of latency.

- Rapid understanding of the topics discussed.

- Duration to go through each topic reduced.

- Outcomes of the meeting sessions improved.

We took a qualitative approach to evaluate the achievement of these goals by surveys, which were handed out after models, created using the CIFE MBS automation tool were integrated and fully used in the on-site meetings.

\section{THE CASE STUDIES}

\subsection{Case Studies}

We tested the CIFE MBS automation tool (Rischmoller et al., 2017) in two case study projects. In both case studies the main researcher was hired as the project BIM coordination consultant, who produced Integrated (or Federated) Project Models (IPMs) aggregating individual models from different sources (i.e., different project stakeholders using a variety of model authoring tools). The project personnel were not aware of the

\footnotetext{
${ }^{5}$ Center for Integrated Facility Engineering
} 
BIM coordinator acting also as a researcher aiming at testing the benefits of utilizing the tool.

The first case study is a multi-billion-dollar power plant megaproject. The second case study is a multi-million-dollar data center project. The main researcher joined both projects during the construction phase, in which individual BIM models had already been created by designers and some fabricators for design and trade coordination. These models were not used at the on-site meetings before the introduction of the IPMs produced by the CIFE MBS automation tool.

Using different combinations of the construction management project breakdown categories (e.g., area, level, discipline, contractor, system, etc.), the CIFE MBS automation tool was used to create IPMs that combined the existing design and fabrication models. Each IPM contains a unique MBS, displayed on the Navisworks ® Selection Tree window, reflecting a certain breakdown structure.

\subsection{IPMs implementation on the case study projects}

Using the CIFE MBS automation tool, different MBSs (reflecting different project breakdown structures) were created and displayed by the BIM coordinator (i.e., main researcher) at the existing daily and weekly coordination and planning meetings (e.g., pull planning meetings and daily subcontractor planning meetings). The BIM coordinator followed the ongoing issue or conversation by displaying on the model the relevant parts of the projects under discussion. The IPMs were displayed on one screen whereas the meeting agendas and/or other type of documents (e.g., graphics and 2D drawings) were shown on another. The main researcher did not provide any notification about the utilization of the IPMs in these meetings, in which most participants had never utilized any $3 \mathrm{D}$ models. This is a lesson learned from previous projects in which attempts of forcing the use of 3D models in these meetings failed when the participants were very conscious of the models and paid less attention to the issues at hand. In these attempts the participants lost interest and patience quickly when a model navigator did not prepare the right viewpoints for an issue and spent time to find the right model contents on the spot.

The variety of modelling capabilities of the designers, fabricators and subcontractors involved in each case study project led to the situation in which not all the digital model files used to assemble the IPMs matched the 2D drawings used for the construction execution. Some models were outdated in comparison to most recent $2 \mathrm{D}$ drawings, while some of the 2D drawings where outdated in comparison to the most current design model files. These however did not prevent the utilization of the IPMs for planning and coordination purposes. Sometimes even 3D placeholders were good enough to improve the quality of the discussion and accelerate issue resolutions in the meetings.

\section{RESULTS}

\subsubsection{IPMs awareness}

After the introduction of the IPMs in different type of on-site meetings in the case study projects, the meeting participants (usually subcontractor's superintendents and managers, but also owner representatives, planners, safety and quality managers, etc.) soon started to realize the "presence" of the IPMs and how the models "followed" the ongoing discussions. This lead the meetings' participants to start shifting their focus to the screen displaying the IPM. After two weeks, the meeting participants realized that they could not only expect the model to "follow" the discussions but that they could actually "require" the model to show parts of the project to support their ongoing discussions. Within another 
two weeks the meeting participants began to request more advanced features of the IPMs such as color-coding certain systems or model elements, color-coding the pieces by plan vs. actual, and overlaying IPMs with other design/construction documents.

Within two months of the use of IPMs the main researcher received request from project engineers, project managers from both the general contractor and the subcontractors, as well as tech-savvy superintendents to learn the use of the IPMs and some basic navigation functions. Quite a few participants also asked for the availability of the IPMs on mobile devices so they could use them in the field. Eventually these participants became the model navigators in the meetings, assisting and facilitating meetings by themselves instead of depending of a BIM coordinator. It was clear that this less intrusive approach of introducing the IPMs to the field personnel paid off.

\subsubsection{Survey}

To further understand the benefits of the use of the IPMs created by the CIFE MBS automation tool, a survey was carried out among the project participants that had attended meetings in which the IPMs were used, in each case study project. Figure 1 and 2 show the composition of the population sampled, and the number of meetings attended by the people surveyed on both cases studies. Figure 3 shows the survey results. The results of the surveys indicate that the experience was overwhelmingly perceived as positive by the project team members of each case study.
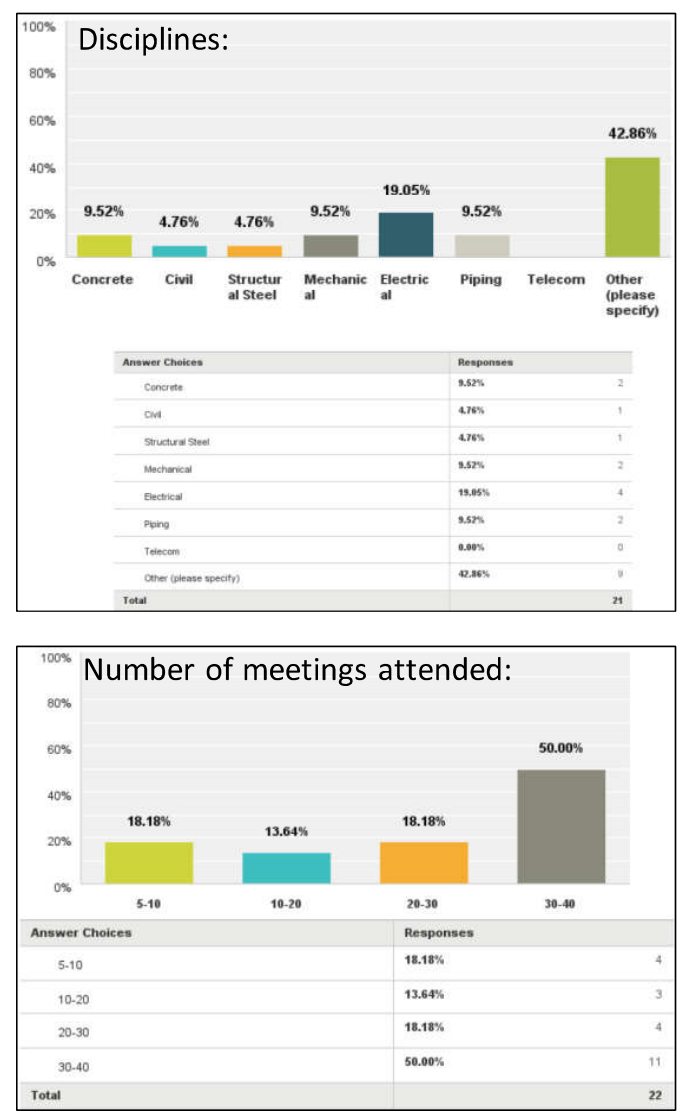

Figure 1: Case study 1 survey demographics 

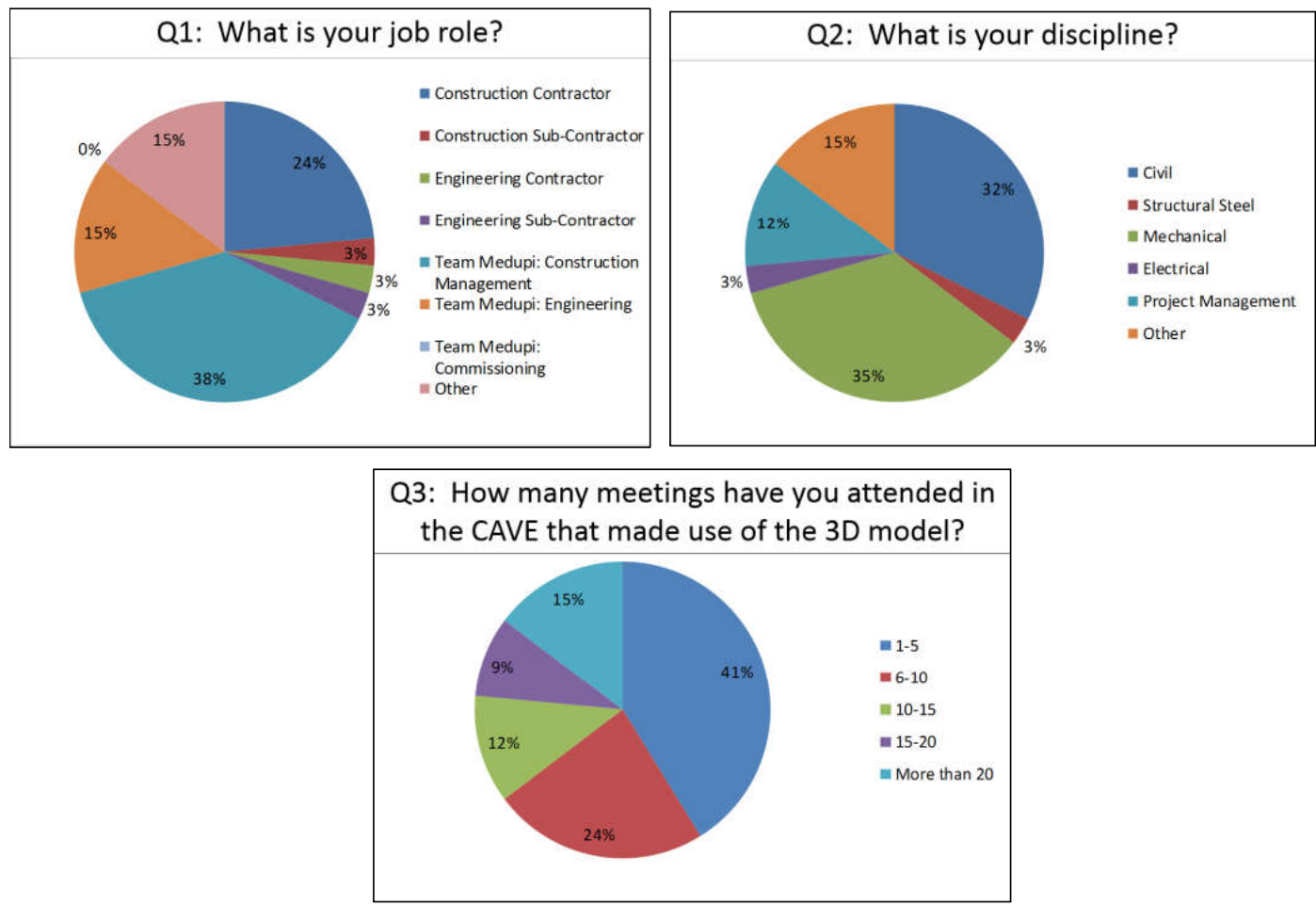

Figure 2: Case study 2 survey demographics

\subsubsection{Reduction of latency and ICE sessions}

We discovered that most of the times a model navigator could answer to a model query in less than fifteen seconds. This strengthened the interaction between the IPMs and the meeting participants, resulting in a reduction of latency during the meetings. With the aid of the IPMs the participants were consistently on the same page when discussing a topic and the time of searching through emails and documents for information was also drastically reduced. Furthermore, the IPMs enabled the participants to ask many more questions earlier (i.e., questions that they wouldn't have thought of until very late during the project execution) because they realized that through the MBS they could rapidly access model, and hence project visualization and information to support discussions during ongoing meetings (Sack et al., 2009), that otherwise would have demanded extra time (and/or another meeting) to gather documents and drawings which in addition do not provide the visualization and rapid access to project information that can be obtained by using the model. As indicated in the survey results the meeting participants perceived that the use of IPMs greatly improved the understanding of the meeting topics as well as the communication, coordination, collaboration, and integration among the participants.

In the first case study the CIFE ICE session (Kunz and Fischer, 2012) principles and ideas were applied in order to support the diverse project stakeholder community to achieve their project objectives through the use of the IPMs in meetings carried out at the Computer Advanced Visualization Environment (CAVE) facility, dedicated for BIMenhanced design and construction coordination.

After the embracement of the IPMs as central part of their meetings by the project team members, the CIFE ICE session methodology was introduced, gradually transforming most of the project meetings into ICE sessions in which the IPMs became just another piece of a more holistic approach to collaboration and integration. The transformation 
involved some planning and setups in advance including the meeting logistics (e.g., stand up meetings, u-shaped chairs distribution, etc.), clear definition of the expected outcomes, pre-planning tasks (e.g., preparation of the IPMs), definition of roles during the ICE sessions (e.g., facilitator, recorder, IPM operator), required resources (e.g., laptops, whiteboard, post-its, and projectors/TVs), as well as the decision-making processes and methods.

Rate the level of achievement for the following elements when using the 3D model in the CAVE:

Q4: Rapid understanding of topics discussed

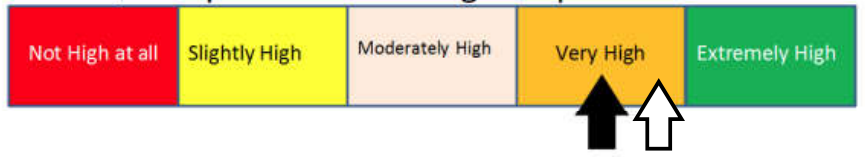

Q5: Improved Communication among participants

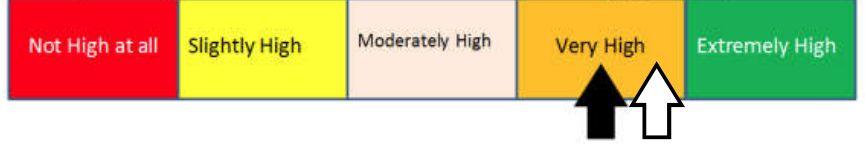

Q6: Improved Coordination among participants

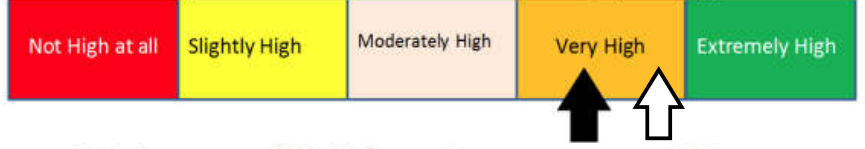

Q7: Improved Collaboration among participants

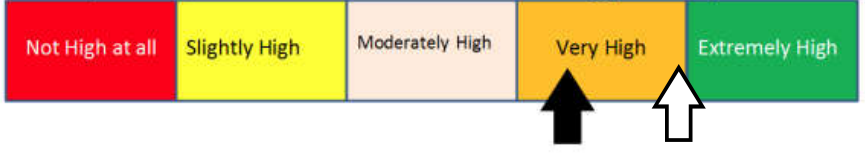

Q8: Improved Integration among participants

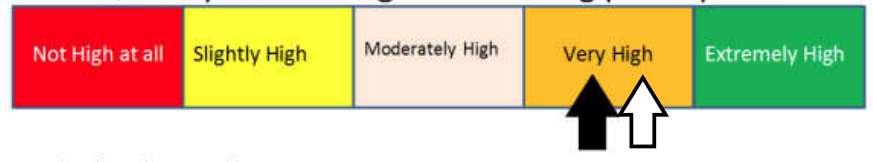

Meeting durations and Outcomes:

\section{Q9: Reduction of meeting duration}

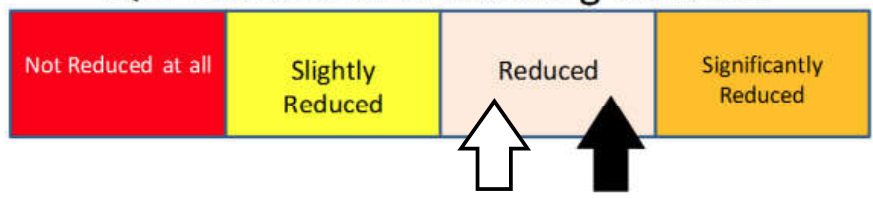

Q10: Improvement of Outcomes

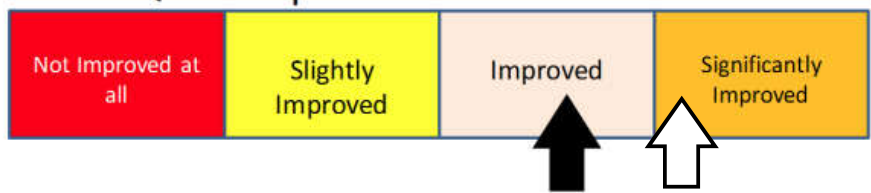

Case Study 1 Case Study 2

Figure 3: Survey questions and results 


\subsubsection{Design Push versus Pull}

On-site meetings focusing on planning, scheduling and tracking of the work sequence, constructability, and coordination; did not necessarily require a high level of geometrical precision from the IPMs, sometimes even placeholders in the complete project 3D environment were good enough to support discussions about construction sequences. However, even a "perfect" model was not always needed for coordination purposes, the lack of quality and completeness in parts of the IPMs was identified by the construction teams and communicated to the design teams. The designers in both case studies were not used to hearing comments about their models from superintendents, project managers, and foremen. However, they embraced the feedback and updated (or completed) their models rapidly without asking for extra payment (under design-build contracts). In some cases, they updated models that they would not have been either persuaded or forced to update during the design stage. The IPM exposed good and bad things about the models to a new audience and exerted peer pressures among the meeting participants, including the designers, who became more willingness to "collaborate" with the people in charge of converting the design information into a physical reality. This is different from the previous efforts carried out during the design and preconstruction stages in which designers and subcontractors were pushed (either convinced or forced) to model in a certain way, using certain criteria and processes (e.g., following a naming convention, signing-off model to freeze the design, etc.), and in a timely manner so that the 2D drawings will always be extracted from the fabrication models. Upstream awareness about IPMs that will be scrutinized downstream by construction professionals and last planners pulled not only higher diligence on the Building Information Modelling efforts, but in the design itself represented now not only in 2D drawings but also in the IPMs.

\section{DISCUSSION}

Instead of windows within model visualization software tools showing data attached to each model component or element in the form of properties, attributes or parameters; the MBS provides access to a different type of model information through "a family tree subdivision of a project beginning with the end objectives and then subdividing these objectives into successively smaller subdivisions" (the same original description of the WBS). Furthermore, the CIFE MBS automation tool provides the ability and flexibility to create rapidly as many different MBSs as needed, eliminating the need to discuss which breakdown structure is better. The idea of "allowing improved project visibility" originally proposed by the WBS concept for the control phase can be expanded to all the project development phases in which IPMs are reviewed using appropriate MBSs. The research presented on this paper is limited to testing the benefits of using IPMs including MBSs created with the CIFE MBS automation tools, used by multidisciplinary audiences of 1030 persons working on complex tasks concurrently in the same room during the project construction stage. In this sense, the benefits of timely availability of information to all development participants, and the reduction of the traditional cycle of working periods and meeting periods, proposed by Concurrent Engineering is fulfilled by using IPMs with appropriate MBSs. On this paper we have explored these benefits specifically during meetings that were subsequently transformed into ICE sessions, in which latency was reduced, and communication, coordination, collaboration, and integration among participants was improved. 


\section{CONCLUSIONS}

Finding out the best way to managing the implementation of BIM is a task that can still be considered under exploration. And considering the complexity associated to the multidisciplinary and multi-phase nature of our projects, as well as new tools, technologies and associated processes appearing every time at a faster pace, it can be stated that managing the implementation of BIM is a task that will probably be under permanent exploration and adjustment. As part of the current BIM management approaches, trying to push BIM into the construction process, if milestone goals of a BIM execution plan are not achieved timely, the project will not wait and the BIM implementation will give up to, either explicitly or implicitly, avoid the unmanageable associated to unfulfilled achieved milestones. When this happens, it is not only the modelling effort which is affected, but the design itself leads the construction management approach to struggle on focusing with managing the unavoidable issues "common" to most of the projects and reflected in documents like RFIs and Change Orders.

The IPMs and MBSs approaches presented in this paper introduce a novel mechanism that can be added to any BIM implementation effort providing a feedback loop from downstream project execution that shall contribute to pull innovative, more simple and effective BIM management approaches.

\section{REFERENCES}

Chachere, J., Kunz, J., and Levitt, R. (2004). Observation, Theory, and Simulation of Integrated Concurrent Engineering: Grounded Theoretical Factors that Enable Radical Project Acceleration, CIFE Working Paper \#WP087, Aug, 2004.

Chachere, J., Kunz, J., and Levitt, R. (2009). The Role of Reduced Latency in Integrated Concurrent Engineering, CIFE Working Paper \#WP116, Aug, 2009.

Coffe, T. (2006). The Future of Integrated Concurrent Enginnering in Spacecraft Design, The Lean Aerospace Initiative Working Paper Series, MIT, 2006

Kunz, J. and Fischer, M. (2012). Virtual Design and Construction; Themes, Case Studies and Implementation Suggestions, CIFE Working Paper \#097, Version 14, Jan, 2012

Rischmoller L., Dong N., Fischer M., and Khanzode A. (2017). Automation of the Building Information Model Breakdown Structure" In: Proc. Lean \& Computing in Construction Congress (LC3), Vol. 3 (IGLC), Heraklion, Greece

Sacks, R., Treckmann, M., and Rozenfeld, O. (2009). Visualization of Workflow to Support Lean Construction. Journal of construction engineering and management, Vol. 135, Issue 12

Smith, J. (1998). "Concurrent Engineering in the Jet Propulsion Laboratory Project Design Center", Society of Automotive Engineers, Long Beach, CA, U.S.A., June 4.

Smith, D., and L. Koenig (1998). "Modeling and Project Development", European Space \& Research Centre, Noordwijk, The Netherlands, November 3.

Tommelein, I. (1998). "Pull-driven scheduling for pipe-spool installation: simulation of a lean construction technique." Journal of construction engineering and management., 124 (4) 279288.

Wall, S. (1999). Reinventing the Design Process: Teams and Models, IAF, Specialist Symposium: Novel Concepts for Smaller, Faster \& Better Space Missions, Redondo Beach, California, USA, April 19-21.

Wall, S. (2000). Design Process Enhancements for Planetary Missions. 4th International Conference on Low Cost Planetary Missions, Maryland, USA, May 2

Wall, S., D. Smith, L. Koenig, and J. Baker (1999). "Team Structures and Processes in the Design of Space Missions", MTG: 1999 IEEE Aerospace Conference, Snowmass at Aspen, CO, U.S.A., March 6-13. 